SLAC-PUB-7681

December, 1997

\title{
Transmission of Supersymmetry Breaking from a 4-Dimensional Boundary
}

\author{
Eugene A. Mirabelli and Michael E. Peskin円 \\ Stanford Linear Accelerator Center \\ Stanford University, Stanford, California 94309 USA
}

\begin{abstract}
In the strong-coupling limit of the heterotic string theory constructed by Hořava and Witten, an 11-dimensional supergravity theory is coupled to matter multiplets confined to 10-dimensional mirror planes. This structure suggests that realistic unification models are obtained, after compactification of 6 dimensions, as theories of 5-dimensional supergravity in an interval, coupling to matter fields on 4-dimensional walls. Supersymmetry breaking may be communicated from one boundary to another by the 5-dimensional fields. In this paper, we study a toy model of this communication in which 5-dimensional super-Yang-Mills theory in the bulk couples to chiral multiplets on the walls. Using the auxiliary fields of the Yang-Mills multiplet, we find a simple algorithm for coupling the bulk and boundary fields. We demonstrate two different mechanisms for generating soft supersymmetry breaking terms in the boundary theory. We also compute the Casimir energy generated by supersymmetry breaking.
\end{abstract}

Submitted to Physical Review D

\footnotetext{
${ }^{1}$ Work supported by the Department of Energy, contract DE-AC03-76SF00515.
} 


\section{Introduction}

In their recent investigation of the structure of strongly-coupled heterotic string theory, Hořava and Witten have introduced a new paradigm for models of unification [1, 2, 3]. To construct the strong-coupling limit of the heterotic string, they began from the 11dimensional strong-coupling limit of the Type IIA string theory. They compactified this model on $S^{1} / Z_{2}$, that is, on an interval of length $\ell$ bounded by mirror (orientifold) planes. They then argued that a 10 -dimensional $E_{8}$ super-Yang-Mills theory appears on each plane. The final structure is a set of two $E_{8}$ gauge theories on the mirror planes, coupling to supergravity in the interior of the interval.

This arrangement had an immediate phenomenological advantage over the weakly coupled $E_{8} \times E_{8}$ heterotic string theory [3]. When $\ell$ was increased, the low-energy value of Newton's constant decreased proportional to $1 / \ell$, while the $E_{8}$ gauge coupling remained fixed. Thus, by adjustment of $\ell$, one could arrange a unification of gauge and gravitational couplings. Choosing a large value of $\ell$ relative to the 11-dimensional Planck scale justified the use of only field-theoretic, and not intrinsically string-theoretic, degrees of freedom. At the same time, Hořava and Witten obtained reasonable values for the gauge and gravitational couplings for values of $\ell$ not so large, in the sense that both of these scales could be considered to be of the order of the grand unification scale of $2 \times 10^{16} \mathrm{GeV}$ inferred from the values of the gauge couplings at low energy.

In a realistic phenomenology, 6 of the transverse 10 dimensions should be compactified. Then one would obtain a 5-dimensional theory on an interval with mirror-plane boundaries. Plausibly, this theory could be described as a 5-dimensional supergravity field theory, perhaps with some additional bulk supermultiplets, coupling to matter supermultiplet fields on the walls. If $\ell$ is the largest dimension in this geometry, it is reasonable that the theory should make sense in the limit in which $\ell$ is taken to be nonzero while the finite size of the 6dimensional compactification space is ignored.

Hořava and Witten introduced another very interesting idea on the nature of these compactifications. They pointed out that the matter theory could be at strong coupling on one boundary, and could break supersymmetry spontaneously there. Then the supersymmetrybreaking effects could be communicated to the other boundary by 11- or 5-dimensional fields. In this way, the theory on one boundary would become the 'hidden sector' for the phenomenological supersymmetry theory on the other boundary.

Hořava tried to make this mechanism of communication explicit by exhibiting a term in the 11-dimensional Lagrangian which coupled the gaugino condensate on the boundary to the 3-form gauge field $C_{A B C}$ of the bulk supergravity theory [4]. He found that this term had a perfect-square structure

$$
\Delta L=-\frac{1}{12 \kappa^{2}} \int d^{11} x\left(\partial_{11} C_{A B C}-\frac{\sqrt{2}}{16 \pi}\left(\frac{\kappa}{4 \pi}\right)^{2 / 3} \bar{\chi} \Gamma_{A B C} \chi \delta\left(x^{11}\right)\right)^{2}
$$

where $\chi$ is the 10 -dimensional gaugino and $8 \pi \kappa^{2}$ is the 11-dimensional Newton constant. 
Hořava argued that, if the gaugino bilinear obtains a nonzero value, there is no solution for $C_{A B C}$ which is consistent with supersymmetry.

Hořava's observations raise two interesting questions of principle. The first concerns the structure of (1). We might want to know how the delta function on the boundary shown in (四) arises. The square of this term integrated over $x^{11}$ gives a factor $\delta(0)$ in the boundary Lagrangian. It is a puzzling issue whether this term is reasonably included in a purely fieldtheoretic description of the Hořava-Witten compactification, or whether the presence of this term implies that any such field-theoretic description is incomplete.

The second question comes from the fact that the communication between the two boundaries comes from the 3-form gauge field, a rather exotic agent. From the general form of the potential energy in supergravity, the 4-dimensional theory which we would obtain by compactifying 6 dimensions and then taking the limit $\ell \rightarrow 0$ must contain a direct coupling of the superpotentials on the two boundaries. We would like to know how this coupling arises, and how much of this coupling is present in the compactified theory before we take the limit $\ell \rightarrow 0$. In the standard approach to supersymmetry breaking mediated by supergravity, this coupling is the source of the soft supersymmetry-breaking mass terms for squarks and sleptons. It would be wonderful if the presence of an extended fifth dimension had specific consequences for the superparticle mass spectrum which could be verified experimentally.

We have tried to find the answers to these questions by studying a toy model in which supergravity is replaced by a Yang-Mills supermultiplet. Consider, then, 5-dimensional superYang-Mills theory on an interval of length $\ell$ bounded by mirror planes, coupled to chiral multiplets $\phi, \phi^{\prime}$ on the 4 -dimensional boundaries. In the limit $\ell \rightarrow 0$, this theory must have a potential energy with the $D$-term contribution

$$
V=\frac{g^{2}}{2}\left(Q \phi^{\dagger} \phi+Q^{\prime} \phi^{\prime \dagger} \phi^{\prime}\right)^{2}
$$

where $g$ is the effective 4-dimensional coupling constant and $Q, Q^{\prime}$ are the gauge charges of $\phi, \phi^{\prime}$. So we can ask in this system also how much of the coupling between boundaries which is required in the limit $\ell \rightarrow 0$ survives when $\ell$ is kept nonzero. The related problem of coupling a 5-dimensional of hypermultiplets to a superpotential on the boundary has been studied previously by Sharpe [5], but, we feel, without giving the insight that we are seeking.

A convenient strategy for coupling 5-dimensional supermultiplets to a 4-dimensional boundary is to work with the off-shell supermultiplets, including auxiliary fields. Under straightforward dimensional reduction, 5-dimensional multiplets reduce to 4-dimensional $N=2$ supermultiplets. A mirror plane, or orientifold, obtained by a $Z_{2}$ identification has lower supersymmetry, and so on such a plane a 5-dimensional multiplet should reduce to a 4-dimensional $N=1$ supermultiplet. Nevertheless, if we have the correct off-shell multiplet, we can couple it straightforwardly to 4-dimensional fields on the boundary.

In Section 2, we will present the necessary formalism for coupling a 5-dimensional superYang-Mills multiplet to an orientifold boundary. We will identify the off-shell 4-dimensional supermultiplet which couples to the boundary fields and use this multiplet to construct 
the 4-dimensional boundary Lagrangian. In Section 3, we will discuss the role of the $\delta(0)$ terms which appear in this Lagrangian, illustrating our conclusions by some explicit oneand two-loop calculations.

In Section 4, we will use the formalism that we have developed to discuss the communication of supersymmetry breaking from one boundary to the other. We will first analyze the case in which supersymmetry is spontaneously broken by a Fayet-Iliopoulos term on one boundary. Then we will present a more involved example in which supersymmetry is communicated by loop diagrams which span the fifth dimension.

If supersymmetry is spontaneously broken, the vacuum energy can be nonzero. In general, the vacuum energy will contain a term, called the Casimir energy [6], which depends on the separation of the two boundaries. In the eventual application to supergravity, this dependence is needed to fix the size of the compact geometry. Though the case of 5-dimensional Yang-Mills theory is simpler than that of supergravity in several respects, it is still interesting to compute the Casimir energy for this case. In Section 5, we evalute this energy for the models of the communication of supersymmetry-breaking studied in Section 4 and note the similarities of the two computations.

In Sections 3 through 5, we will be carrying out weak-coupling perturbation theory computations in the nonrenormalizable 5-dimensional Yang-Mills theory. Our attitude toward this nonrenormalizability is a pragmatic one; we will be pleased if quantities of physical interest turn out to be ultraviolet-finite at the leading order of perturbation theory. That will be true in our explicit calculations of the scalar mass term and the Casimir energy. Presumably, the higher-order corrections to these computations are cutoff-dependent and are regulated by the underlying string theory. In this paper, we will not be concerned with effects beyond the leading nontrivial order.

Finally, in Section 6, we will discuss the relation of our formalism to Hořava's analysis and give an explanation of the coupling shown in (四).

Our approach to the Hořava-Witten theory complements the many attempts to understand the structure of this theory by direct analysis of the 11-dimensional Lagrangian. General properties of the strong-coupling limit of the heterotic string theory have been discussed in [7, 8, 9]. More explicit studies of the compactification of the Hořava-Witten theory have been discussed recently by several groups. Some of these analysis [10, 11, 12, 13, 14] have emphasized the connection to the venerable mechanism of supersymmetry breaking in string theory by $E_{8}$ gaugino condensation [15, while others [16, 17] have relied on the ScherkSchwarz mechanism [18] in the bulk to provide a new source of supersymmetry breaking. Brax and Turok [19] have contributed an observation on the possibility of large hierarchies in the 5-dimensional geometry, if all of the relevant 5-dimensional fields can be made massive. We hope that the methods of analysis that we introduce here, when generalized to supergravity, will clarify the many possible sources of supersymmetry breaking which may contribute in the Hořava-Witten approach to unification. 


\section{Bulk and boundary supermultiplets}

In this section, we will set up the formalism for coupling 5-dimensional super-Yang-Mills theory to an orientifold boundary. The 5-dimensional Yang-Mills multiplet contains a vector field $A^{M}$, a real scalar field $\Phi$, a gaugino $\lambda^{i}$.

In this paper, capitalized indices $M, N$ run over $0,1,2,3,5$, lower-case indices $m$ run over $0,1,2,3$, and $i, a$ are internal $S U(2)$ spinor and vector indices, with $i=1,2, a=1,2,3$. We use a timelike metric $\eta_{M N}=\operatorname{diag}(1,-1,-1,-1,-1)$ and take the following basis for the Dirac matrices:

$$
\gamma^{M}=\left(\left(\begin{array}{cc}
0 & \sigma^{m} \\
\bar{\sigma}^{m} & 0
\end{array}\right),\left(\begin{array}{cc}
-i & 0 \\
0 & i
\end{array}\right)\right)
$$

where $\sigma^{m}=(1, \vec{\sigma}), \bar{\sigma}^{m}=(1,-\vec{\sigma})$. Though it is conventional in the literature to use raised and lowered spinor indices, we find it less confusing to write out explicitly the $2 \times 2$ and $4 \times 4$ charge conjugation matrices

$$
c=-i \sigma^{2}, \quad C=\left(\begin{array}{cc}
c & 0 \\
0 & c
\end{array}\right) .
$$

In 5-dimensional supersymmetry, it is convenient to rewrite 4-component Dirac spinors as symplectic-Majorana spinors, Dirac fermions which carry an $S U(2)$ spinor index and satisfy the constraints

$$
\psi^{i}=c^{i j} C \bar{\psi}^{j T} .
$$

A symplectic-Majorana spinor can be decomposed into 4-dimensional chiral spinors according to

$$
\psi^{i}=\left(\begin{array}{c}
\phi_{L}^{i} \\
\phi_{R}^{i}
\end{array}\right)
$$

where $\phi_{L, R}^{i}$ are two-component spinors connected by

$$
\phi_{L}^{i}=c^{i j} c \phi_{R}^{j *}, \quad \phi_{R}^{i}=c^{i j} c \phi_{L}^{j *} .
$$

Symplectic-Majorana spinors $\psi^{i}, \chi^{i}$ satisfy the identity

$$
\bar{\psi}^{i} \gamma^{M} \cdots \gamma^{P} \chi^{j}=-c^{i k} c^{j \ell} \bar{\chi}^{\ell} \gamma^{P} \cdots \gamma^{M} \psi^{k}
$$

including the minus sign from fermion interchange.

In this notation, the 5-dimensional Yang-Mills multiplet is extended to an off-shell multiplet by adding an $S U(2)$ triplet $X^{a}$ of real-vauled auxiliary fields [26]. Write the members of the multiplet as matrices in the adjoint representation of the gauge group: $A^{M}=A^{M A} t^{A}$, etc. The the supersymmetry transformation laws are

$$
\begin{aligned}
\delta_{\xi} A^{M} & =i \bar{\xi}^{i} \gamma^{M} \lambda^{i} \\
\delta_{\xi} \Phi & =i \bar{\xi}^{i} \lambda^{i} \\
\delta_{\xi} \lambda^{i} & =\left(\sigma^{M N} F_{M N}-\gamma^{M} D_{M} \Phi\right) \xi^{i}-i\left(X^{a} \sigma^{a}\right)^{i j} \xi^{j} \\
\delta_{\xi} X^{a} & =\bar{\xi}^{i}\left(\sigma^{a}\right)^{i j} \gamma^{M} D_{M} \lambda^{j}-i\left[\Phi, \bar{\xi}^{i}\left(\sigma^{a}\right)^{i j} \lambda^{j}\right]
\end{aligned}
$$


where the symplectic-Majorana spinor $\xi^{i}$ is the supersymmetry parameter, $D_{M} \Phi \equiv \partial_{M} \Phi-$ $i\left[A_{M}, \Phi\right]$ (and similarly for $D_{M} \lambda$ ), and $\sigma^{M N} \equiv \frac{1}{4}\left[\gamma^{M}, \gamma^{N}\right]$.

Now we would like to project this structure down to a 4-dimensional $N=1$ supersymmetry transformation acting on fields on the orientifold wall. In a field theory description, an orientifold at $x^{5}=0$ is described by imposing the boundary condition on bulk fields $a\left(x, x^{5}\right)$

$$
a\left(x^{m}, x^{5}\right)=P a\left(x^{m},-x^{5}\right)
$$

where $P$ is an intrinsic parity equal to \pm 1 . The quantum number $P$ must be assigned to fields in such a way that it leaves the bulk Lagrangian invariant. Then fields of $P=-1$ vanish on the walls but have nonvanishing derivatives $\partial_{5} a$. Note that, since $A^{5}$ vanishes on the boundary, $\partial_{5}=D_{5}$ on the boundary and $\partial_{5} a$ is gauge-covariant.

Let $\xi_{L}^{1}$ be the supersymmetry parameter of the $N=1$ supersymmetry transformation on the wall. Then the $P$ assignments of the fields in the bulk supermultiplet are determined as follows:

\begin{tabular}{|c|c|c|} 
& $P=+1$ & $P=-1$ \\
\hline \hline$\xi$ & $\xi_{L}^{1}$ & $\xi_{L}^{2}$ \\
\hline$A^{M}$ & $A^{m}$ & $A^{5}$ \\
\hline$\Phi$ & - & $\Phi$ \\
\hline$\lambda^{i}$ & $\lambda_{L}^{1}$ & $\lambda_{L}^{2}$ \\
\hline$X^{a}$ & $X^{3}$ & $X^{1,2}$ \\
\hline
\end{tabular}

On the wall at $x^{5}=0$, the supersymmetry transformation (9) reduces to the following transformation of the even-parity states generated by $\xi_{L}^{1}$ :

$$
\begin{aligned}
\delta_{\xi} A^{m} & =i \xi_{L}^{1 \dagger} \bar{\sigma}^{m} \lambda_{L}^{1}-i \lambda_{L}^{1 \dagger} \bar{\sigma}^{m} \xi_{L}^{1} \\
\delta_{\xi} \lambda_{L}^{1} & =\sigma^{m n} F_{m n} \xi_{L}^{1}-i\left(X^{3}-\partial_{5} \Phi\right) \xi_{L}^{1} \\
\delta_{\xi} X^{3} & =\xi_{L}^{1 \dagger} \bar{\sigma}^{m} D_{m} \lambda_{L}^{1}-i \xi_{L}^{1 \dagger} c \partial_{5} \lambda_{L}^{2 *}+h . c . \\
\delta_{\xi} \partial_{5} \Phi & =-i \xi_{L}^{1 T} c \partial_{5} \lambda_{L}^{2}-i \xi_{L}^{1 \dagger} c \partial_{5} \lambda_{L}^{2 *} .
\end{aligned}
$$

The last two equations imply

$$
\delta_{\xi}\left(X^{3}-\partial_{5} \Phi\right)=\xi_{L}^{1 \dagger} \bar{\sigma}^{m} D_{m} \lambda_{L}^{1}+\text { h.c. . }
$$

These are just the transformation laws for an $N=14$-dimensional vector multiplet [27], with $A^{m}, \lambda_{L}^{1}$, and $\left(X^{3}-\partial_{5} \Phi\right)$ transforming as the vector, gaugino, and auxiliary D fields.

The appearance of the quantity $\partial_{5} \Phi$ in the auxiliary field should not be a surprise. It is the expectation value of this quantity that breaks supersymmetry in Scherk-Schwarz mechanism [18]. Thus, $\partial_{5} \Phi$ should appear in the order parameter of supersymmetry breaking.

Now it is obvious how to couple the 5 -dimensional gauge multiplet to 4-dimensional chiral multiplets on the boundary. We write the Lagrangian as

$$
S=\int d^{5} x\left\{\mathcal{L}_{5}+\sum_{i} \delta\left(x^{5}-x_{i}^{5}\right) \mathcal{L}_{4 i}\right\},
$$


where the sum includes the walls at $x_{i}^{5}=0, \ell$. The bulk Lagrangian should be the standard one for a 5-dimensional super-Yang-Mills multiplet,

$$
\begin{aligned}
\mathcal{L}_{5}= & \frac{1}{g^{2}}\left(-\frac{1}{2} \operatorname{tr}\left(F_{M N}\right)^{2}+\operatorname{tr}\left(D_{M} \Phi\right)^{2}+\operatorname{tr}\left(\bar{\lambda} i \gamma^{M} D_{M} \lambda\right)\right. \\
& \left.+\operatorname{tr}\left(X^{a}\right)^{2}-\operatorname{tr}(\bar{\lambda}[\Phi, \lambda])\right),
\end{aligned}
$$

with $\operatorname{tr}\left[t^{A} t^{B}\right]=\delta^{A B} / 2$. The bulk fields should be constrained to satisfy the boundary conditions (10) at the walls. Since the supersymmetry generated by $\xi_{L}^{1}$ relates fields with the same boundary conditions, this $N=1$ supersymmetry is an invariance of (15) under the constraint.

The boundary Lagrangian should have the standard form of a four-dimensional chiral model built from supermultiplets $\left(\phi, \psi_{L}, F\right)$. Here and in the rest of the paper, it is important to distinguish boundary chiral scalar fields, which we will label by $\phi$, from the bulk scalar field $\Phi$. The explicit form of this boundary Lagrangian is

$$
\begin{aligned}
\mathcal{L}_{4}= & D_{m} \phi^{\dagger} D^{m} \phi+\psi_{L}^{\dagger} i \bar{\sigma}^{m} D_{m} \psi_{L}+F^{\dagger} F \\
& -\sqrt{2} i\left(\phi^{\dagger} \lambda_{L}^{T} c \psi_{L}+\psi^{\dagger} c \lambda_{L}^{*} \phi\right)+\phi^{\dagger} D \phi,
\end{aligned}
$$

with $D_{m}=\left(\partial_{m}-i A_{m}\right)$, and with with the gauge fields $\left(A_{m}, \lambda_{L}, D\right)$ replaced by the boundary values of the bulk fields $\left(A_{m}, \lambda_{L}^{1}, X^{3}-\partial_{5} \Phi\right)$. The boundary Lagrangian $\mathcal{L}_{4}$ is invariant by itself under an $N=1$ supersymmetry transformation of the boundary fields and the supersymmetry transformation (12) of the bulk fields. Thus, the complete action (14) is $N=1$ supersymmetric.

In principle, we could add to (14) additional terms involving a four-dimensional integral of the boundary values of the vector fields, or terms coupling the chiral fields to higher $\partial_{5}$ derivatives of the vector fields at the boundary. These terms would correspond to contributions that are more singular at the boundary that the ones we have considered. For our present purposes, we only point out that these terms are not necessary for supersymmetry, and we neglect them from here on. We will show in explicit calculations that the terms we have written suffice to give amplitudes which converge to the correct 4-dimensional limits as $\ell \rightarrow 0$.

With the action (14), the boundary scalar field $\phi$ at $x^{5}=0$ couples to the auxiliary field $X^{3}$ through the terms

$$
\int d^{5} x\left\{\frac{1}{g^{2}} \operatorname{tr}\left(X^{3}\right)^{2}+\delta\left(x^{5}\right) \phi^{\dagger}\left(X^{3}-\partial_{5} \Phi\right) \phi\right\} .
$$

The field $\Phi$ is a dynamical field in the interior, but $X^{3}$ is an auxiliary field and may be integrated out. This gives a boundary Lagrangian of the form

$$
\int d^{4} x\left\{-\phi^{\dagger}\left(\partial_{5} \Phi\right) \phi-\frac{1}{2}\left(\phi^{\dagger} t^{A} \phi\right)^{2} \delta(0)\right\} .
$$




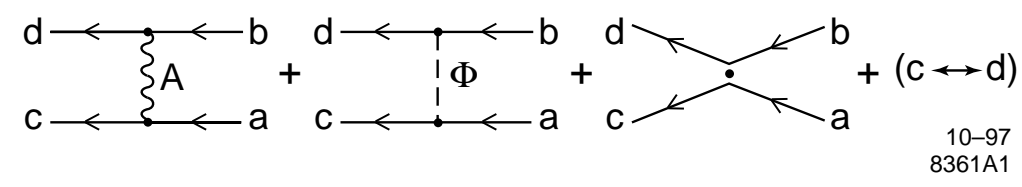

Figure 1: Feynman diagrams contributing to the scattering process $\phi \phi \rightarrow \phi \phi$.

Thus, our formalism does contain singular terms proportional to $\delta(0)$ on the boundary, which arise naturally from integrating out the auxiliary fields. In principle, the complete description of the orientifold wall in string theory could contain additional couplings involving higher derivatives $\partial_{5}$ of the bulk fields and representing a finite thickness and a shape of the wall. However, the Lagrangian we have written, with the $\delta(0)$ but no additional singular terms, is a completely self-consistent supersymmetric system.

\section{$3 \quad$ Bulk and boundary perturbation theory}

In the previous section, we have found that singular terms proportional to $\delta(0)$ on the boundary arise naturally when bulk and boundary fields are coupled supersymmetrically. What is still unclear is whether these terms can lead to sensible results when one performs computations in this theory, or whether these terms signal the breakdown of a purely fieldtheoretic description. We believe that these singular terms do make sense at the field theory level. Their role is to provide counterterms which are necessary in explicit calculations to maintain supersymmetry. In this section, we will illustrate this conclusion with some explicit calculations in perturbation theory.

As a first, simplest, example, consider the scattering amplitude for scalars on a wall. The Feynman diagrams contributing to the process $\phi_{a}+\phi_{b} \rightarrow \phi_{c}+\phi_{d}$ are shown in Figure 1 . The propagator of a free massless bulk field is

$$
\left\langle a\left(x, x^{5}\right) a\left(y, y^{5}\right)\right\rangle=\int_{k 5} \frac{i}{k^{2}-\left(k^{5}\right)^{2}} e^{-i k \cdot(x-y)}\left(e^{i k^{5}\left(x^{5}-y^{5}\right)}+P e^{i k^{5}\left(x^{5}+y^{5}\right)}\right),
$$

where

$$
\int_{k 5}=\int \frac{d^{4} k}{(2 \pi)^{4}} \frac{1}{2 \ell} \sum_{k^{5}}
$$

with $k^{5}$ summed over the values $\pi m / \ell, m=$ integer. Here and in the rest of our discussion, $k$ represents a the 4 -dimensional momentum components of $k^{M}$.

The sum of diagrams in Figure 1 is given by

$$
i \mathcal{M}\left(\phi_{a}+\phi_{b} \rightarrow \phi_{c}+\phi_{d}\right)=-i g^{2} t_{c a}^{A} t_{d b}^{A}\left(\frac{1}{2 \ell} \sum_{k^{5}} \frac{\left(k^{5}\right)^{2}}{\left(p_{c}-p_{a}\right)^{2}-\left(k^{5}\right)^{2}}+\delta(0)\right.
$$




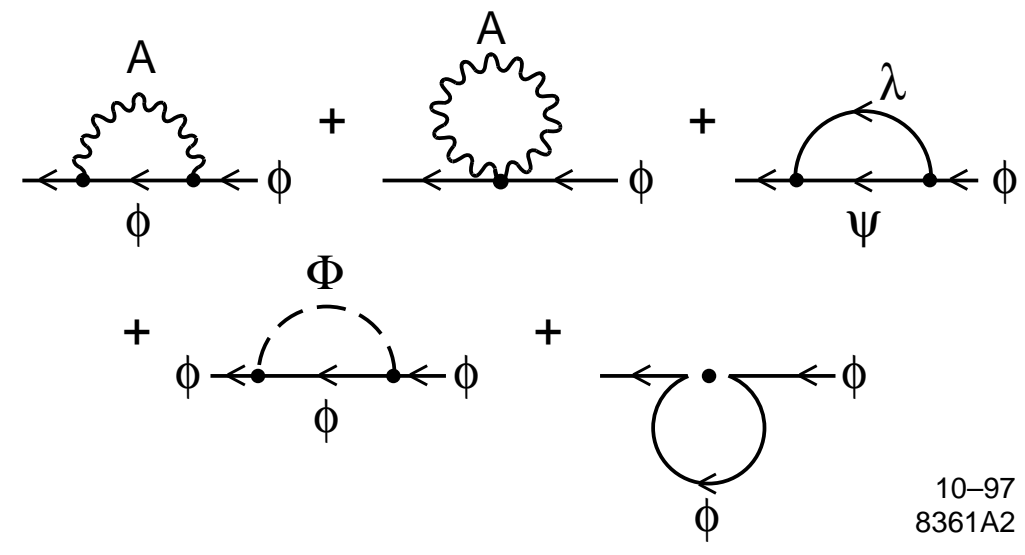

Figure 2: Feynman diagrams contributing to the $\phi$ self-energy at one-loop order.

$$
\left.-\frac{1}{2 \ell} \sum_{k^{5}} \frac{\left(p_{c}+p_{a}\right) \cdot\left(p_{d}+p_{b}\right)}{\left(p_{c}-p_{a}\right)^{2}-\left(k^{5}\right)^{2}}\right)+(c \leftrightarrow d) .
$$

If we represent

$$
\delta(0)=\frac{1}{2 \ell} \sum_{k^{5}} 1=\frac{1}{2 \ell} \sum_{k^{5}} \frac{k^{2}-\left(k^{5}\right)^{2}}{k^{2}-\left(k^{5}\right)^{2}},
$$

the first two terms have a neat cancellation and we find the finite result

$$
\begin{aligned}
i \mathcal{M}\left(\phi_{a}+\phi_{b} \rightarrow \phi_{c}+\phi_{d}\right) & =-i g^{2} t_{c a}^{A} t_{d b}^{A}\left(\frac{1}{2 \ell} \sum_{k^{5}} \frac{\left(p_{c}-p_{a}\right)^{2}-\left(p_{c}+p_{a}\right) \cdot\left(p_{d}+p_{b}\right)}{\left(p_{c}-p_{a}\right)^{2}-\left(k^{5}\right)^{2}}\right)+(c \leftrightarrow d) \\
& =-i g^{2} t_{c a}^{A} t_{d b}^{A}\left(\frac{1}{2 \ell} \sum_{k^{5}} \frac{-2 u}{t-\left(k^{5}\right)^{2}}\right)+(c \leftrightarrow d) .
\end{aligned}
$$

If $\ell \rightarrow 0$ with the dimensionless coupling $g^{2} / \ell$ fixed, the terms with $k^{5} \neq 0$ become negligible. Then we recover the scalar particle scattering amplitude of a 4-dimensional $N=1$ supersymmetric gauge theory.

As a second example, consider the self-energy of the scalar $\phi$, computed at the one-loop level. By supersymmetry, the $\phi$ cannot obtain a mass in perturbation theory, but it is interesting to see explicitly how the cancellation occurs. The Feynman diagrams for the $\phi$ self-energy are shown in Figure 2. The first four diagrams all involve one field that propagates in four dimensions and one field that propagates in the fifth dimension. Thus, if $p$ is the external 4-momentum, all of these diagrams will have the structure

$$
-i M^{2}\left(p^{2}\right)=g^{2} t^{A} t^{A} \int_{k 5} \frac{1}{k^{2}-\left(k^{5}\right)^{2}} \frac{1}{(p-k)^{2}} N\left(k, k^{5}, p\right),
$$



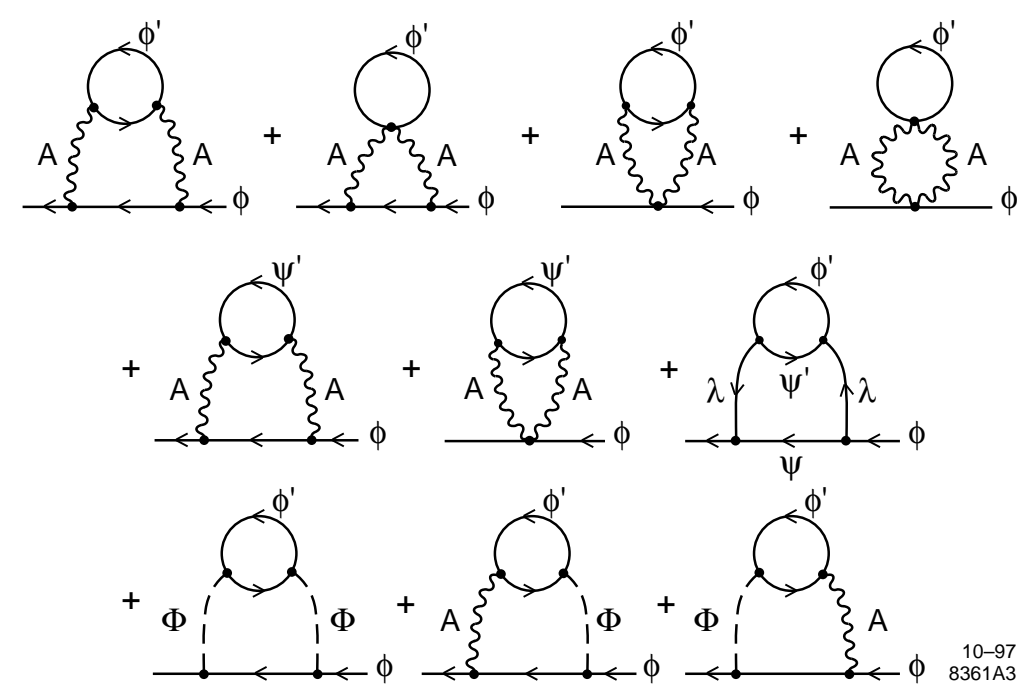

Figure 3: Feynman diagrams contributing to the mass shift of a scalar $\phi$ on one wall due to loop effects of the supermultiplet on the other wall.

where $N$ is a polynomial in momenta. Using the representation (22), we can bring the last diagram into this form as well. Then the five diagrams give contributions

$$
\begin{aligned}
N & =-(2 p-k)^{2}+4(p-k)^{2}-4 k \cdot(k-p)+\left(k^{5}\right)^{2}+\left(k^{2}-\left(k^{5}\right)^{2}\right) \\
& =0
\end{aligned}
$$

Here the $\delta(0)$ term enters quite explicitly as a counterterm which cancels the singluar behavior of the $\Phi$ exchange diagram and thus allows the complete cancellation required by supersymmetry.

To prepare for the next section, it will be useful to illustrate one more cancellation required by supersymmetry. Consider the renormalization of the mass of a scalar $\phi$ on one wall due to loop diagrams involving the supermultiplet on the other wall. This mass shift is given by the sum of the two-loop diagrams shown in Figure 3. In our discussion of these diagrams, we will assign the chiral fields $\phi$ at $x^{5}=0$ to the representation $R$ of the gauge group, and we will assign the fields $\phi^{\prime}$ at $x^{5}=\ell$ to the representation $R^{\prime}$.

The diagrams of Figure 3 form a gauge-invariant set. We might characterize them as the diagrams of order $g^{4} N$, where $N$ is the number of matter multiplets on the second wall. Thus, by supersymmetry, their sum must vanish.

It is not difficult to see this explicitly. Since we are interested in the shift of the $\phi$ mass term, we can set the external momentum equal to zero. Then let $q$ be the loop momentum of the matter loop on the wall at $x^{5}=\ell$, Let $\left(k, k^{5}\right)$ and $\left(k, \hat{k}^{5}\right)$ be the momenta carried by the two propagators of the gauge multiplet that connect the two walls. These momenta are 
quantized, with

$$
k^{5}=\pi n / \ell, \quad \hat{k}^{5}=\pi \hat{n} / \ell,
$$

but the integers $n$ and $\hat{n}$ are summed over independently, since $k^{5}$ is not conserved in the interaction of bulk fields with the walls. Then all of the diagrams shown in Figure 3 can be written in the form

$$
-i M^{2}=i g^{4} C_{2}(R) C\left(R^{\prime}\right) \int_{q} \int_{k 55} \frac{N\left(k, k^{5}, \hat{k}^{5}, q\right)}{\left(k^{2}\right)\left(k^{2}-\left(k^{5}\right)^{2}\right)\left(k^{2}-\left(\hat{k}^{5}\right)^{2}\right)\left(q^{2}\right)\left((q-k)^{2}\right)},
$$

where $N$ is a polynomial in momenta, $C\left(R^{\prime}\right) \delta^{A B}=\operatorname{tr}_{R^{\prime}}\left[t^{A} t^{B}\right]$ is the sum over the gauge quantum numbers of the multiplet at $x^{5}=\ell$, the integral over $q$ is a simple 4-dimensional momentum integral, and

$$
\int_{k 55}=\int \frac{d^{4} k}{(2 \pi)^{4}} \frac{1}{2 \ell} \sum_{n} \frac{1}{2 \ell} \sum_{\hat{n}} 4 \cdot(-1)^{n+\hat{n}} .
$$

This expression includes the $k^{5}$-dependence of the propagators, obtained by evaluating (19) at $x^{5}=0, y^{5}=\ell$.

To see that the diagrams of this set must cancel, it is easiest to compare this calculation to the corresponding two-loop mass renormalization in four dimensions. The diagrams on the first two lines of Figure 3 contain, from the five-dimensional gauge multiplet, only the propagators of the fields $A_{m}$ and $\lambda_{L}^{1}$ which appear in a 4-dimensional gauge multiplet. Thus, their contributions to the numerator polynomial $N$ are exactly those of the corresponding diagrams in 4 dimensions. To treat the last three diagrams, we note the identity

$$
0=\frac{1}{2 \ell} \sum_{k^{5}}(-1)^{n}=\frac{1}{2 \ell} \sum_{k^{5}}(-1)^{n} \frac{k^{2}-\left(k^{5}\right)^{2}}{k^{2}-\left(k^{5}\right)^{2}},
$$

since the second term is a representation of $\delta\left(x^{5}\right)$ evaluated at $x^{5}=\ell$. Each $\Phi$ propagator comes with a factor $\left(k^{5}\right)^{2}$, due to the couplings (18) at each wall. The identity (29) allows us to replace this $\left(k^{5}\right)^{2}$ by $k^{2}$. Then each diagram gives the same contribution to the numerator as the corresponding 4-dimensional diagram with the $\Phi$ replaced by a $D$-term interaction. Thus, the numerator polynomial $N$ turns out to be exactly the one that appears in the 4-dimensional calculation.

At this point, we know that the integral (27) must vanish. It is not difficult to evaluate the various contributions to the numerator and to see that they cancel. In the Appendix, we give a formula for the numerator factor $N$ from which this can be verified explicitly.

\section{$4 \quad$ Wall to Wall Supersymmetry Breaking}

We have now described and tested an explicit form for the coupling of 4-dimensional supermultiplets on the boundary to gauge supermultiplets in the bulk. Now we can use this 
formalism to see how supersymmetry breaking on one wall is communicated to the other wall to provide soft supersymmetry-breaking terms. In this section, we will give two examples of such communication, one through a direct tree-level coupling and the other induced by loop effects.

The simplest example of the communication of supersymmetry breaking is obtained in a theory in which the wall at $x^{5}=\ell$ contains no boundary matter fields. We choose the gauge group to be $U(1)$ and write a a Fayet-Iliopoulos $D$ term on this boundary. With the identification of the $D$ term given in Section 2, the following boundary action preserves $N=1$ supersymmetry:

$$
\mathcal{L}_{4}=\kappa\left(X^{3}-\partial_{5} \Phi\right)
$$

Integrating out the auxiliary field $X^{3}$ leads to a $\delta(0)$ term which is an irrelevant constant. The dynamical $\Phi$ field is affected by this term, in a manner that we can compute from the action

$$
S=\int d^{5} x\left\{\frac{1}{2 g^{2}}\left(\partial_{M} \Phi\right)^{2}-\kappa \partial_{5} \Phi \delta\left(x^{5}-\ell\right)\right\} .
$$

Varying this action with respect to $\Phi$, we find that the Fayet-Iliopoulos term induces a background expectation value of $\Phi$ which depends only on $x^{5}$ and satisfies the equation

$$
\frac{1}{g^{2}} \partial_{5}^{2}\langle\Phi\rangle+\kappa \partial_{5} \delta\left(x^{5}-\ell\right)=0
$$

In solving this equation, we should remember that the geometry with mirror planes arose from a identification of points in a compactification of $x^{5}$ on a circle. Thus, $\left\langle\Phi\left(x^{5}\right)\right\rangle$ must be a periodic function of $x^{5}$ with period $2 \ell$ and so $\partial_{5} \Phi$ must integrate to zero around the circle. This boundary condition requires that we choose as the solution to (32)

$$
\partial_{5}\langle\Phi\rangle=-g^{2} \kappa\left(\delta\left(x^{5}-\ell\right)-\frac{1}{2 \ell}\right) .
$$

Inserting this result into the $D$-term coupling on the wall at $x^{5}=0$, given by (17), we find a scalar mass term given by

$$
M_{\phi}^{2}=g^{2} Q \frac{\kappa}{2 \ell},
$$

where $Q$ is the $U(1)$ charge of the scalar field, with no corresponding mass term induced for the fermions on the wall. If the dynamics on the wall at $x^{5}=\ell$ gives a $D$-term of fixed magnitude there, the $\Phi$ field transfers this across the fifth dimension to create a soft scalar mass term on the wall at $x^{5}=0$.

One subtlety of the Fayet-Iliopoulos mechanism of supersymmetry breaking is that the symmetry breaking $D$ term can sometimes be compensated by shifting the vacuum expectations value of a scalar field. We can see a similar possibility here. Generalize the previous model to include several chiral multiplets $\phi_{i}$ on the wall at $x^{5}=0$, and additional chiral multiplets $\phi_{j}$ on the wall at $x^{5}=\ell$. (As always, it is important to distinguish between the boundary scalar fields $\phi$ and the bulk field $\Phi$.) Assign these multiplets the charges $Q_{i}, Q_{j}$ 
under the $U(1)$ symmetry. In the most general situation, all of the scalar fields might acquire vacuum expectation values. Then the Lagrangian for $\Phi$ will take the form

$$
\begin{aligned}
S= & \int d^{5} x\left\{\frac{1}{2 g^{2}}\left(\left(X^{3}\right)^{2}+\left(\partial_{M} \Phi\right)^{2}\right)+\left(\sum_{i} Q_{i} \phi_{i}^{\dagger} \phi_{i}\right)\left(X^{3}-\partial_{5} \Phi\right) \delta\left(x^{5}\right)\right. \\
& \left.+\left(\kappa+\sum_{j} Q_{j} \phi_{j}^{\dagger} \phi_{j}\right)\left(X^{3}-\partial_{5} \Phi\right) \delta\left(x^{5}-\ell\right)\right\} .
\end{aligned}
$$

For simplicity, we assume that the $\phi_{i}$ and $\phi_{j}$ are represented only by vacuum expectation values that are independent of position on the walls. Then varying the action (35) with respect to $\Phi$ gives an equation analogous to (32) whose solution is

$$
\partial_{5}\langle\Phi\rangle=-g^{2}\left[\left(\sum_{i} Q_{i} \phi_{i}^{\dagger} \phi_{i}\right)\left(\delta\left(x^{5}\right)-\frac{1}{2 \ell}\right)+\left(\kappa+\sum_{j} Q_{j} \phi_{j}^{\dagger} \phi_{j}\right)\left(\delta\left(x^{5}-\ell\right)-\frac{1}{2 \ell}\right)\right] .
$$

This result reduces to (33) when we turn off the expection values of the $\phi_{i}$ and $\phi_{j}$. If we insert this expression into (35), and also integrate out the auxiliary field $X^{3}$, the various $\delta(0)$ terms cancel, leaving behind

$$
S=\int d^{5} x\left\{-\frac{g^{2}}{4 \ell}\left(\kappa+\sum_{i} Q_{i} \phi_{i}^{\dagger} \phi_{i}+\sum_{j} Q_{j} \phi_{j}^{\dagger} \phi_{j}\right)^{2}\right\}
$$

To minimize the vacuum energy, we must set the various vacuum expectation values to the supersymmetric condition

$$
\kappa+\sum_{i} Q_{i} \phi_{i}^{\dagger} \phi_{i}+\sum_{j} Q_{j} \phi_{j}^{\dagger} \phi_{j}=0
$$

if this is possible.

If the supersymmetric theory on the wall at $x^{5}=\ell$ breaks supersymmetry spontaneously without inducing a $D$ term, it is necessary to go to a higher order in perturbation theory to find the supersymmetry-breaking communication. If supersymmetry breaking causes a mass splitting among chiral supermultiplets on the wall at $x^{5}=\ell$, and these multiplets couple to the gauge field in the bulk, then the diagrams shown in Figure 3 induce a supersymmetrybreaking mass for scalars on the wall at $x^{5}=0$. Since, in the scheme we are studying, the particle number of a chiral multiplet at $x^{5}=0$ is conserved, this is the only soft supersymmetry-breaking term that can be generated.

The generation of the scalar mass term in this example is very similar to that in 'gaugemediated' 4-dimensional models of supersymmetry breaking [20]. The same set of diagrams appears, with only the difference that our gauge fields live in 5 dimensions.

To illustrate the computation of these diagrams, we study the simplest multiplet which appears in models of gauge-mediation. We introduce on the wall at $x^{5}=\ell$ two chiral superfields $\left(\phi^{\prime}, \bar{\phi}^{\prime}\right)$ which transform under the gauge group as a vectorlike representation 
$\left(R^{\prime}+\bar{R}^{\prime}\right)$. (Recall that we are using $R$ to denote representation of the chiral fields $\phi$ at $\ell=0$.) We give this multiplet a supersymmetric mass $m$ and induce an additional mass term for the scalar fields from the vacumm expectation value of an $F$-term. Then the fermions have a Dirac mass $m$, while the bosons have a (mass) ${ }^{2}$ matrix

$$
M^{2}\left(\begin{array}{c}
\phi^{\prime} \\
\phi^{* \prime}
\end{array}\right)=\left(\begin{array}{cc}
m^{2} & m^{2} x \\
m^{2} x & m^{2}
\end{array}\right)\left(\frac{\phi^{\prime}}{\phi^{* \prime}}\right) \text {. }
$$

The eigenvectors of this matrix are species $\phi_{+}^{\prime}, \phi_{-}^{\prime}$ in the representation $R^{\prime}$. Thus, we find the mass spectrum on the wall at $x^{5}=\ell$,

$$
m^{2}\left(\phi_{+}^{\prime}\right)=m_{+}^{2}, \quad m^{2}\left(\phi_{-}^{\prime}\right)=m_{-}^{2}, \quad m^{2}\left(\psi^{\prime}\right)=m^{2},
$$

with $m_{ \pm}^{2}=m^{2}(1 \pm x)$. This spectrum satisfies $\operatorname{str}\left[M^{2}\right]=0$. Our calculation will follow closely the discussion of gauge-mediated scalar masses in this model given by Martin [21]. It is straightforward to generalize our calculation to models of supersymmetry breaking with nonvanishing supertrace. However, in that case, the scalar masses induced by gaugemediation are cutoff-dependent even in 4 dimensions [22]. Similar divergences appear also in the 5-dimensional case.

To compute the scalar mass, we repeat the calculation of the digrams in Figure 3, now assigning to the particles in the loop the mass spectrum described in the previous paragraph. As in the previous section, the identity (29) can be used to replace $\left(k^{5}\right)^{2}$ by $k^{2}$ in the numerator of the diagrams with $\Phi$ exchange. Then the result reduces to a sum of two-loop scalar integrals, just as in the 4-dimensional case.

To write the result precisely, define [23]

$$
\left(m_{1} m_{2}\left|m_{3}\right| m_{4}\right)=\int \frac{d^{d} k}{(2 \pi)^{d}} \int \frac{d^{d} q}{(2 \pi)^{d}} \frac{1}{k^{2}+m_{1}^{2}} \frac{1}{k^{2}+m_{2}^{2}} \frac{1}{q^{2}+m_{3}^{2}} \frac{1}{(q-k)^{2}+m_{4}^{2}}
$$

to be the Euclidean (Wick-rotated) scalar two-loop integral with four propagators, and denote Euclidean scalar integrals with additional or fewer propagators by brackets with more or fewer labels $m_{i}$. In our calculation, $k^{5}$ is summed over the values $\pi n / \ell$; denote the sum in (28) as

$$
[\mathcal{A}]=\frac{1}{2 \ell} \sum_{n} \frac{1}{2 \ell} \sum_{\hat{n}} 4 \cdot(-1)^{n+\hat{n}} \mathcal{A}\left(k^{5}, \hat{k}^{5}\right) .
$$

The basic scalar integral shown in Figure 4 is then written

$$
\left[\left(k^{5} \hat{k}^{5}\left|m_{1}\right| m_{2}\right)\right] \text {. }
$$

Finally, though a term with $k^{2}$ in the numerator can be reduced to scalar integrals, it is more convenient to retain this factor under the integral in (41). By abuse of notation, we will write a term with $k^{2}$ in the numerator as, for example, $\left[k^{2}\left(k^{5} \hat{k}^{5}\left|m_{2}\right| m_{3}\right)\right]$.

Using the notation, the scalar mass due to the diagrams of Figure 3 is

$$
m_{\phi}^{2}=-g^{4} C_{2}(R) C\left(R^{\prime}\right)\left[k^{2} \mathcal{R}+4 \mathcal{S}\right]
$$




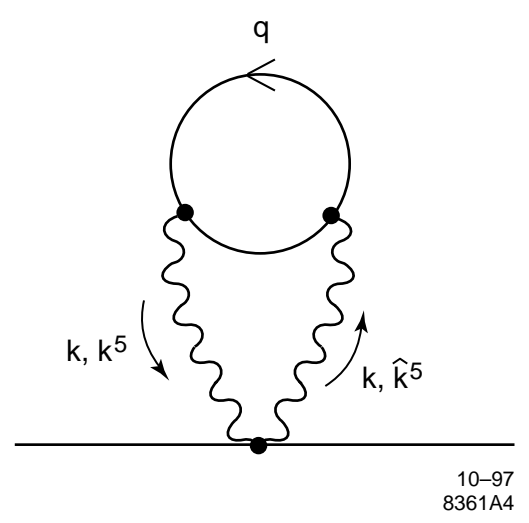

Figure 4: The basic integral which appears in the two-loop contribution to the scalar field mass.

where

$$
\begin{aligned}
\mathcal{R}= & \left(k^{5} \hat{k}^{5}\left|m_{+}\right| m_{+}\right)+\left(k^{5} \hat{k}^{5}\left|m_{-}\right| m_{-}\right)+2\left(k^{5} \hat{k}^{5}\left|m_{+}\right| m_{-}\right)+4\left(k^{5} \hat{k}^{5}|m| m\right) \\
& -4\left(k^{5} \hat{k}^{5}\left|m_{+}\right| m\right)-4\left(k^{5} \hat{k}^{5}\left|m_{-}\right| m\right) \\
\mathcal{S}= & m_{+}^{2}\left\{\left(k^{5} \hat{k}^{5}\left|m_{+}\right| m_{+}\right)-\left(k^{5} \hat{k}^{5}\left|m_{+}\right| m\right)\right\}-m^{2}\left\{\left(k^{5} \hat{k}^{5}|m| m\right)-\left(k^{5} \hat{k}^{5}\left|m_{+}\right| m\right)\right\} \\
& +m_{-}^{2}\left\{\left(k^{5} \hat{k}^{5}\left|m_{-}\right| m_{-}\right)-\left(k^{5} \hat{k}^{5}\left|m_{-}\right| m\right)\right\}-m^{2}\left\{\left(k^{5} \hat{k}^{5}|m| m\right)-\left(k^{5} \hat{k}^{5}\left|m_{-}\right| m\right)\right\}
\end{aligned}
$$

This expression is full of cancellations which reflect the fact that the answer vanishes when the mass spectrum is supersymmetric. To evaluate this answer more explicitly, we must perform the sums over $k^{5}$ and $\hat{k}^{5}$ and then carry out the two four-dimensional integrals.

The sums can be performed conveniently using a standard trick from finite temperature field theory. Write a contour integral representation

$$
\frac{1}{2 \ell} \sum_{n} 2(-1)^{n} \frac{1}{k^{2}+\left(k^{5}\right)^{2}}=\oint \frac{d k^{5}}{2 \pi} \frac{2 e^{i k^{5} \ell}}{e^{2 i k^{5} \ell}-1} \frac{1}{k^{2}+\left(k^{5}\right)^{2}},
$$

where the contour encloses the poles of the integrand at $k^{5}=\pi n / \ell$. Draw the contour as a line from left to right just below the real axis and another line from right to left just above this axis. Push the first line down and pick up the pole at $k^{5}=-i k$; push the second line up and pick up the pole at $k^{5}=i k$. We find two identical contributions which sum to

$$
\frac{1}{k} \frac{1}{\sinh k \ell}
$$

This manipulation can be performed separately on each of the propagators joining the two walls. 
At the same time, the scalar integrals over the momentum $q$ can be evaluated explicitly. Define the function $b\left(k^{2}, m_{1}^{2}, m_{2}^{2}\right)$ by

$$
\int \frac{d^{d} q}{(2 \pi)^{d}} \frac{1}{q^{2}+m_{1}^{2}} \frac{1}{(q-k)^{2}+m_{2}^{2}}=\frac{1}{(4 \pi)^{d}}\left\{\frac{2}{\epsilon}-\gamma-b\left(k^{2}, m_{1}^{2}, m_{2}^{2}\right)+\mathcal{O}(\epsilon)\right\}
$$

for $d=4-\epsilon$. When we evaluate the loop integrals on the wall in (45), the divergent terms cancel and we are left with differences of these scalar functions,

$$
\begin{aligned}
\mathcal{R} \rightarrow & R\left(k^{2}\right)=b\left(k^{2}, m_{+}^{2}, m_{+}^{2}\right)+b\left(k^{2}, m_{-}^{2}, m_{-}^{2}\right)+2 b\left(k^{2}, m_{+}^{2}, m_{-}^{2}\right)+4 b\left(k^{2}, m^{2}, m^{2}\right) \\
& -4 b\left(k^{2}, m_{+}^{2}, m^{2}\right)-4 b\left(k^{2}, m_{-}^{2}, m^{2}\right) \\
\mathcal{S} \rightarrow & S\left(k^{2}\right)=m_{+}^{2}\left\{b\left(k^{2}, m_{+}^{2}, m_{+}^{2}\right)-b\left(k^{2}, m_{+}^{2}, m^{2}\right)\right\}-m^{2}\left\{b\left(k^{2}, m^{2}, m^{2}\right)-b\left(k^{2}, m_{+}^{2}, m^{2}\right)\right\} \\
& +m_{-}^{2}\left\{b\left(k^{2}, m_{-}^{2}, m_{-}^{2}\right)-b\left(k^{2}, m_{-}^{2}, m^{2}\right)\right\}-m^{2}\left\{b\left(k^{2}, m^{2}, m^{2}\right)-b\left(k^{2}, m_{-}^{2}, m^{2}\right)\right\} .
\end{aligned}
$$

If we then define

$$
P\left(k^{2}\right)=k^{2} R\left(k^{2}\right)+4 S\left(k^{2}\right),
$$

then the combination of these two tricks brings (44) into the form of an integral over $k$. Since this integral is spherically symmetric, we can replace $d^{4} k=2 \pi^{2} d k k^{3}$ and write (44) as

$$
m_{\phi}^{2}=2\left(\frac{g^{2}}{(4 \pi)^{2}}\right)^{2} C_{2}(R) C\left(R^{\prime}\right) \int_{0}^{\infty} d k k \frac{1}{\sinh ^{2} k \ell} P\left(k^{2}\right) .
$$

The function $P\left(k^{2}\right)$ is elementary, and it is not difficult to work out its asymptotic behavior for large and for small $k^{2}$. We present these formulae in the Appendix. It is relevant that $P\left(k^{2}\right) \sim k^{2}$ as $k^{2} \rightarrow 0$, so that $P\left(k^{2}\right)$ is a field-strength renormalization [24] (as the notation is meant to suggest). As $k^{2} \rightarrow \infty, P\left(k^{2}\right) \sim \log \left(k^{2} / m^{2}\right) / k^{2}$. With this information, one can work out the asymptotic behaviors of $m_{\phi}^{2}$.

For small $\ell$, we might expect to go back the the 4-dimensional situation. Formally, taking $\ell \rightarrow 0$ in (47) turns this expression into

$$
\frac{2}{2 \ell} \frac{1}{k^{2}}
$$

which is the $k^{5}=0$ term in the sum (46). Using the explicit asymptotic behavior of $P\left(k^{2}\right)$, we can see that the integral (51) remains well-defined in this limit. Thus, the manipulation is permitted and the result for $m_{\phi}^{2}$ becomes just the 4-dimensional gauge-mediation result with the replacement

$$
\left(\frac{g^{2}}{(4 \pi)^{2}}\right)^{2} \rightarrow\left(\frac{g^{2}}{(4 \pi)^{2}}\right)^{2} \frac{1}{\ell^{2}}
$$

We will write out this result explicitly below. Note that $g^{2} / \ell$ is the effective 4 -dimensional gauge coupling obtained by simple dimensional redution. 


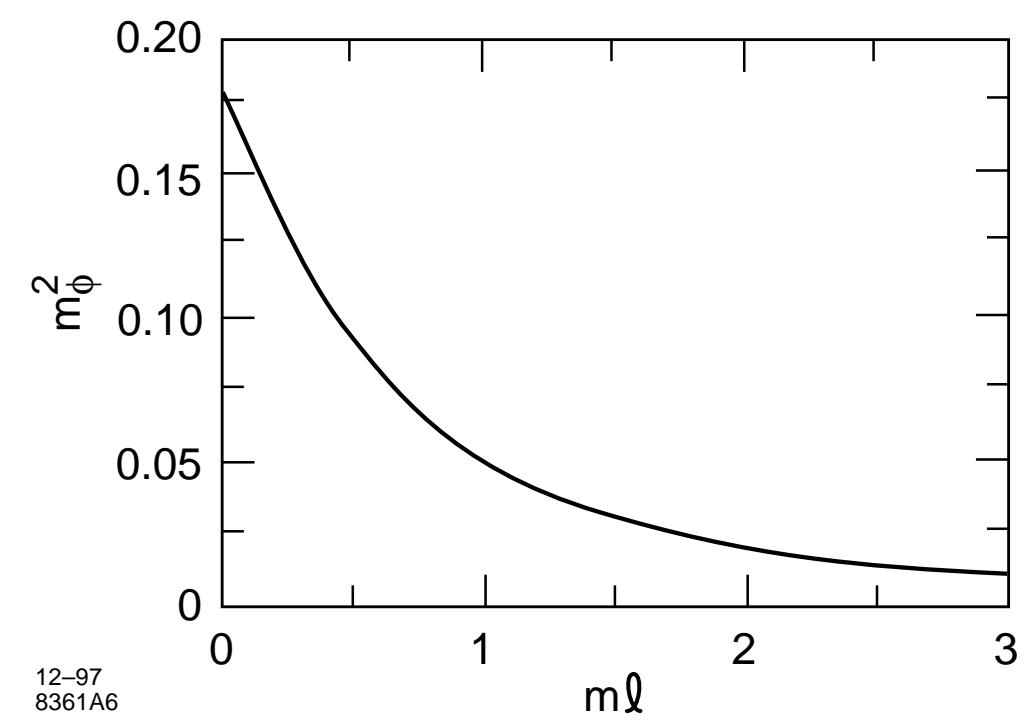

Figure 5: Behavior of the induced supersymmetry breaking mass for scalars at $x^{5}=0$ as a function of $\ell$. We plot $m_{\phi}^{2}$ in units of $\left.\left.2 C_{2}(R) C\left(R^{\prime}\right)\left(g^{2} /(4 \pi)^{2}\right)^{2}\right) \cdot\left(m^{2} / \ell^{2}\right)\right)$.

Another way to derive this result is to show that, for $\ell \rightarrow 0$, all terms in the sum over $k^{5}$ and $\hat{k}^{5}$ are explicitly suppressed by the factor $\ell^{2}$ except for the term with $k^{5}=\hat{k}^{5}=0$. Again, the asymptotic behavior $P\left(k^{2}\right) \sim 1 / k^{2}$ is necessary to complete this argument.

For large $\ell$. the hyperbolic sine in the denomination of (51) cuts off the integrand at very small $k$. Thus, we can find the asymptotic behavior by replacing $P\left(k^{2}\right)$ by its leading term for small $k^{2}$, which is proportional to $k^{2}$. This gives a result proportional to

$$
\left(\frac{g^{2}}{(4 \pi)^{2}}\right)^{2} \frac{1}{\ell^{4}}
$$

Working out all of the details (with the help of some formulae from the Appendix), we find, as $m \ell \rightarrow 0$, the 4-dimensional form [21, 25]

$$
\begin{aligned}
m_{\phi}^{2}= & 2 C_{2}(R) C\left(R^{\prime}\right)\left(\frac{g^{2}}{(4 \pi)^{2}}\right)^{2} \cdot \frac{m^{2}}{\ell^{2}} \\
& \cdot\left\{2(1+x)\left[\log (1+x)-2 \operatorname{Li}_{2}\left(\frac{x}{1+x}\right)+\frac{1}{2} \operatorname{Li}_{2}\left(\frac{2 x}{1+x}\right)\right]+(x \leftrightarrow-x)\right\},
\end{aligned}
$$

where $\operatorname{Li}_{2}(x)$ is the dilogarithm, and, as $m \ell \rightarrow \infty$,

$$
\begin{aligned}
m_{\phi}^{2}= & 2 C_{2}(R) C\left(R^{\prime}\right)\left(\frac{g^{2}}{(4 \pi)^{2}}\right)^{2} \cdot \frac{1}{\ell^{4}} \cdot \zeta(3) \\
& \cdot\left\{\frac{3}{2}\left[\frac{4+x-2 x^{2}}{x^{2}} \log (1+x)-\frac{4-x}{x}\right]+(x \leftrightarrow-x)\right\} .
\end{aligned}
$$


In both of these expressions, the quantity in brackets tends to $x^{2}$ as $x \rightarrow 0$. We see that the induced soft supersymmetry breaking mass term crosses over from the 4-dimensional behavior to a smaller functional form as $\ell$ becomes larger than $1 / m$. In Figure 5, we graph the form of the mass term as a function of $\ell$ for the illustrative value $x=0.3$.

There is another way to understand the behavior of the scalar mass term for $m \ell$ large. If $m$ is large, we can consider the inner loop in Figure to contract to a point. More precisely, because the function $P\left(k^{2}\right)$ is proportional to $k^{2}$ when $k$ is small, this loop gives the pointlike operator $\left(-\nabla^{2}\right)$ acting on the two propagators which run from one wall to the other. The remaining one-loop integral may be evaluated in Euclidean coordinate space. There is one small subtlety to note. The representation of (19) in Euclidean coordinate space is

$$
\begin{aligned}
\left\langle a\left(x, x^{5}\right) a\left(y, y^{5}\right)\right\rangle= & \frac{1}{8 \pi^{2}} \sum_{m}\left(\frac{1}{\left[(x-y)^{2}+\left(x^{5}-y^{5}+2 m \ell\right)^{2}\right]^{3 / 2}}\right. \\
& \left.+P \frac{1}{\left[(x-y)^{2}+\left(x^{5}+y^{5}+2 m \ell\right)^{2}\right]^{3 / 2}}\right)
\end{aligned}
$$

with $m$ summed over all integers. When this expression is evaluated with one end at $x^{5}=\ell$ and the other at $y^{5}=0$, we find (for $P=+1$ )

$$
\langle a(x, \ell) a(0,0)\rangle=\frac{1}{8 \pi^{2}} \sum_{m} \frac{2}{\left[x^{2}+(2 m+1)^{2} \ell^{2}\right]^{3 / 2}} .
$$

Then the evaluation of $m_{\phi}^{2}$ involves the expression

$$
I=\sum_{m, \hat{m}} \int d^{4} x \frac{2}{8 \pi^{2}\left[x^{2}+(2 m+1)^{2} \ell^{2}\right]^{3 / 2}}\left(-\nabla^{2}\right) \frac{2}{8 \pi^{2}\left[x^{2}+(2 \hat{m}+1)^{2} \ell^{2}\right]^{3 / 2}},
$$

containing two propagators which run from a point 0 on the wall at $x^{5}=0$ to a point $x$ on the wall at $x^{5}=\ell$. By combining the two denominators with a Feynman parameter, it is not difficult to do the integral explicitly and then sum over $m$ and $\hat{m}$. The result is

$$
I=\frac{3}{16 \pi^{2}} \zeta(3) \frac{1}{\ell^{4}}
$$

Multiplying this by the coefficient of $k^{2}$ in $P\left(k^{2}\right)$, we find again the result (56). This presentation explains the physical origin of the $1 / \ell^{4}$ behavior of the diagrams.

\section{Casimir energy}

At the same time that supersymmetry breaking on the wall at $x^{5}=\ell$ induces soft supersymmetry-breaking terms in other parts of the theory, it also creates a nonzero vacuum energy. We are particularly interested in the part of this energy which depends on $\ell$ - the Casimir energy [6]-since this term will eventually form a part of the balance which determines 


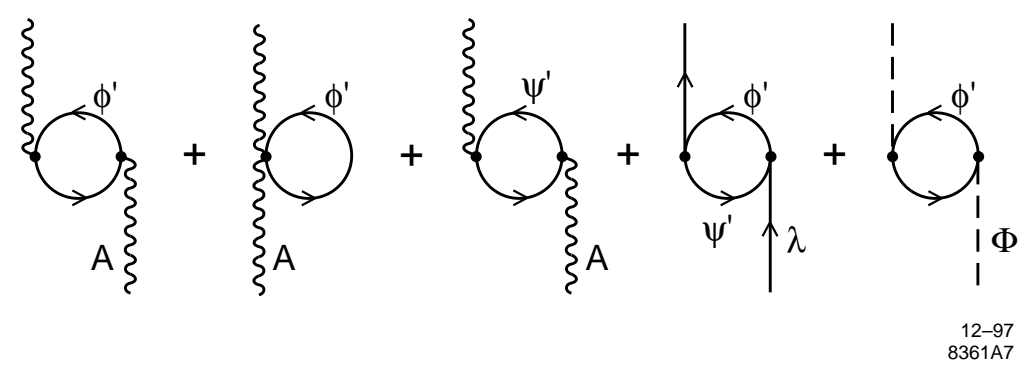

Figure 6: Feynman diagrams contributing to the Casimir energy due to loop effects of the supermultiplet on the wall at $x^{5}=\ell$.

the physical value of $\ell$. In this section, we will compute the Casimir energy due to the two mechanisms of supersymmetry breaking discussed in the previous section. We find it interesting that these calculations run almost in parallel to the calculations of the induced scalar mass term.

Consider first the case of a Fayet-Iliopoulos $D$-term on the boundary at $x^{5}=\ell$. The coupling of this term to the bulk fields is described by the Lagrangian (31), plus a term proportional to $\delta(0)$ resulting from integrating out $X^{3}$. Since (31) is quadratic in $\Phi$, we can integrate this field out explicitly. Using the propagator (33), the coupling of $\Phi$ to the boundary leads to

$$
S_{e f f}=\int d^{5} x \delta\left(x^{5}-\ell\right) \cdot \frac{1}{2} \kappa\left(-\frac{g^{2} \kappa}{2 \ell}\right),
$$

plus an $\ell$-independent term proportional to $\delta(0)$. Thus, we find for the Casimir energy per 4-dimensional volume,

$$
E_{C} / V_{4}=+\frac{g^{2} \kappa^{2}}{4 \ell}
$$

If there are $D$-terms on both boundaries, or if the fields $\phi_{i}$ on the two boundaries obtain expectation values as in (35), the sum of the two $D$ terms appears in place of $\kappa$ in (62). If the two $D$ terms are equal and opposite, the Casimir energy vanishes. Also, as we observed already in (37), the $\delta(0)$ terms from integrating out $X^{3}$ and $\Phi$ precisely cancel. Thus, in this case, the vacuum energy remains just at zero, as expected from the supersymmetry of the situation.

In the case in which the spectrum at $x^{5}=\ell$ violates supersymmetry but there is no induced $D$ term, the Casimir energy must be generated by radiative corrections. The leading contribution comes from the diagrams shown in Figure 6. These diagrams involve a closed loop on the boundary at $x^{5}=\ell$ and a propagator from the 5-dimensional Yang-Mills theory which winds around the compactified direction.

Though perhaps it is not completely obvious from the beginning, the structure of the diagrams in Figure 6 is very similar to that of the diagrams in Figure 3. As in the previous 
section, we will describe the calculation for the case $\operatorname{str}\left[M^{2}\right]=0$.

In the diagrams of Figure 6, we have only one sum over $k^{5}$. Thus, define for this section

$$
[\mathcal{B}]=\frac{1}{2 \ell} \sum_{n} \mathcal{B}\left(k^{5}\right) .
$$

Then the Casimir energy resulting from this set of diagrams can be written in terms of Euclidean scalar two-loop integrals as

$$
E_{C} / V_{4}=\frac{1}{2} g^{2} d_{G} C\left(R^{\prime}\right)\left[k^{2} \mathcal{R}_{\mathcal{C}}+4 \mathcal{S}_{\mathcal{C}}\right],
$$

where $d_{G} C\left(R^{\prime}\right)=\operatorname{tr}_{R^{\prime}}\left[t^{A} t^{A}\right]$, and

$$
\begin{aligned}
\mathcal{R}_{\mathcal{C}}= & \left(k^{5}\left|m_{+}\right| m_{+}\right)+\left(k^{5}\left|m_{-}\right| m_{-}\right)+2\left(k^{5}\left|m_{+}\right| m_{-}\right)+4\left(k^{5}|m| m\right) \\
& -4\left(k^{5}\left|m_{+}\right| m\right)-4\left(k^{5}\left|m_{-}\right| m\right) \\
\mathcal{S}_{\mathcal{C}}= & m_{+}^{2}\left\{\left(k^{5}\left|m_{+}\right| m_{+}\right)-\left(k^{5}\left|m_{+}\right| m\right)\right\}-m^{2}\left\{\left(k^{5}|m| m\right)-\left(k^{5}\left|m_{+}\right| m\right)\right\} \\
& m_{-}^{2}\left\{\left(k^{5}\left|m_{-}\right| m_{-}\right)-\left(k^{5}\left|m_{-}\right| m\right)\right\}-m^{2}\left\{\left(k^{5}|m| m\right)-\left(k^{5}\left|m_{-}\right| m\right)\right\} .
\end{aligned}
$$

The inner loop of each of these two-loop integrals can be evaluated explicitly, giving the same functions $R\left(k^{2}\right), S\left(k^{2}\right), P\left(k^{2}\right)$ that we saw earlier in (49) and (50).

Again we can simplify the sum over $k^{5}$ using the contour trick from finite temperature field theory. Write

$$
\frac{1}{2 \ell} \sum_{n} \frac{1}{k^{2}+\left(k^{5}\right)^{2}}=\oint \frac{d k^{5}}{2 \pi} \frac{1}{e^{2 i k^{5} \ell}-1} \frac{1}{k^{2}+\left(k^{5}\right)^{2}},
$$

where the contour encloses the poles of the integrand at $k^{5}=\pi n / \ell$. Draw the contour as a line from left to right just below the real axis and another line from right to left just above this axis. Push the first line down and pick up the pole at $k^{5}=-i k$. For the contour integral on the line above the axis, replace

$$
\frac{1}{e^{2 i k^{5} \ell}-1}=-1-\frac{1}{e^{-2 i k^{5} \ell}-1} .
$$

In the second term, push the contour up and pick up the pole at $k^{5}=i k$. These manipulations convert (66) to the form

$$
\frac{1}{k} \frac{1}{e^{2 k \ell}-1}+\int_{-\infty}^{\infty} \frac{d k^{5}}{(2 \pi)} .
$$

The second term in (68) is independent of $\ell$; it represents the contribution to the vacuum energy of the 4-dimensional wall in an infinite 5-dimensional volume. Equivalently, from the point of view of propagators in coordinate space, this term is the contribution of the propagators that go from the wall back to the wall without winding around $x^{5}$. In any event, this term does not contribute to the Casimir energy, and we may drop it. 
After these manipulations, the Casimir energy (64) takes the form

$$
E_{C} / V_{4}=-\frac{1}{2}\left(\frac{g^{2}}{(4 \pi)^{4}}\right) d_{G} C\left(R^{\prime}\right) \int_{0}^{\infty} d k k^{2} \frac{1}{e^{2 k \ell}-1} P\left(k^{2}\right),
$$

where $P\left(k^{2}\right)$ is the same field strength renormalization function that appeared in (51).

As in the previous section, we can analyze the two-loop integral in the limits of small and large $\ell$. Consider first the limit $\ell \rightarrow 0$. If we formally take the limit of small $\ell$ in (69), we obtain a divergent integral

$$
E_{C} / V_{4} \sim-\int_{0}^{\infty} d k \frac{k}{\ell} \frac{1}{k^{2}} \log k^{2}
$$

Thus, unlike the case of $m_{\phi}^{2}$, the contributions to the Casimir energy are dominated by large values of $k^{2}$. The estimate

$$
\int_{0}^{\infty} d k k^{2} \frac{1}{e^{2 k \ell}-1} \frac{1}{k^{2}} \log k^{2} \sim \frac{1}{2 \ell} \log ^{2} \frac{1}{m \ell}
$$

and the asymptotic formula for $P\left(k^{2}\right)$ given in the Appendix gives a precise formula for the small $\ell$ behavior:

$$
E_{C} / V_{4}=-\frac{1}{2}\left(\frac{g^{2}}{(4 \pi)^{4}}\right) d_{G} C\left(R^{\prime}\right) \cdot \frac{4 m^{4} x^{2}}{\ell} \log ^{2} \frac{1}{m \ell} .
$$

For large $\ell$, the analysis can be done along the same lines as those we used for $m_{\phi}^{2}$. The denominator of (69) cuts off the integrand for small $k^{2}$. Thus, we can replace $P(k)$ by its leading term as $k^{2} \rightarrow 0$. With this approximation, the integral is easily evaluated, and we obtain

$$
\begin{aligned}
E_{C} / V_{4}= & -\frac{1}{2}\left(\frac{g^{2}}{(4 \pi)^{4}}\right) d_{G} C\left(R^{\prime}\right) \zeta(5) \frac{1}{\ell^{5}} \\
& \left\{\frac{3}{2}\left[\frac{4+x-2 x^{2}}{x^{2}} \log (1+x)-\frac{4-x}{x}\right]+(x \leftrightarrow-x)\right\} .
\end{aligned}
$$

Comparing (72) and (73), we see the same crossover that we found previously from (55) to (56). In Figure [7, we graph the form of the Casimir energy as a function of $\ell$ for the illustrative value $x=0.3$.

As in the previous section, the behavior of the Casimir energy for large $\ell$ is studied most easily in Euclidean coordinate space. If $\ell$ or $m$ is large, the inner loop of each two-loop diagram can be contracted to a local operator proportional to $\left(-\nabla^{2}\right)$. Then the Casimir energy is proportional to an expression in which this operator acts on a propagator which runs around the compact direction. More specifically, the Casimir energy is proportional to

$$
J=\left.\sum_{m}\left(-\nabla^{2}\right) \frac{1}{8 \pi^{2}\left(x^{2}+(m \ell)^{2}\right)^{3 / 2}}\right|_{x=0}
$$




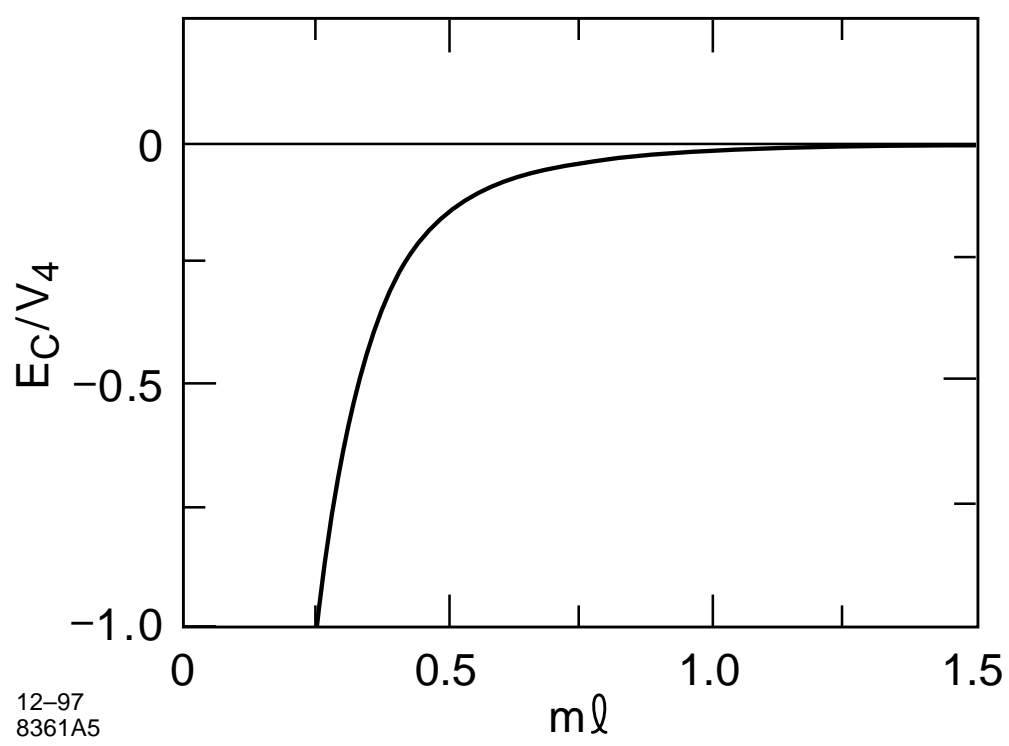

Figure 7: Behavior of the Casimir energy as a function of $\ell$. We plot $\left(E_{C} / V_{4}\right)$ in units of $\left.\frac{1}{2} d_{G} C\left(R^{\prime}\right)\left(g^{2} /(4 \pi)^{4}\right)\right)$.

where the sum over $m$ runs over all integers except $m=0$. This expression evaluates to

$$
J=\frac{3}{32 \pi^{2}} \zeta(5) \frac{1}{\ell^{5}} .
$$

Multiplying this by the coefficient of $k^{2}$ in $P\left(k^{2}\right)$, we return to the result (73).

Both of the individual contributions to the Casimir energy that we found in this section are monotonic in $\ell$. We find it interesting, though, that these two contributions have opposite signs. Thus, it is possible that, in a realistic theory, we could find balancing contributions to the Casimir energy that stabilize the value of $\ell$ at a nonzero value.

\section{Hořava's supersymmetry-breaking structure}

Now that we have analyzed mechanisms for supersymmetry breaking in our toy model, it is interesting to ask whether this sheds light on the mechanism of supersymmetry breaking in string theory proposed by Hořava [ [ reduction of Hořava's system from 11 to 5 dimensions, taking the compact 6 dimensions to be a Calabi-Yau manifold. Under this reduction, the boundary gaugino condensate becomes a 4-dimensional scalar gaugino bilinear on the boundary. The relevant components of the 3 -form gauge field in the bulk are those that multiply the the $(3,0)$ or $(0,3)$ forms of the Calabi-Yau space,

$$
C_{A B C}\left(x, x^{5}, y\right)=c\left(x, x^{5}\right) \Omega_{A B C}(y)+\cdots
$$


These components form two complex 5-dimensional fields which belong to a hypermultiplet in the bulk. Thus, we can try to recover Hořava's coupling of the bulk and boundary fields by considering the coupling of a hypermultiplet in the bulk to chiral fields on the boundary.

We can analyze this problem using arguments similar to those in Section 2. The fivedimensional hypermultiplet consists of a pair of complex scalars $A^{i}$, a Dirac fermion $\chi$, and a pair of complex auxiliary fields $F^{i}$. Under supersymmetry they transform as follows [26]:

$$
\begin{aligned}
\delta_{\xi} A^{i} & =-\sqrt{2} \epsilon^{i j} \bar{\xi}^{j} \chi \\
\delta_{\xi} \chi & =+\sqrt{2} i \gamma^{M} \partial_{M} A^{i} \epsilon^{i j} \xi^{j}+\sqrt{2} F^{i} \xi^{i} \\
\delta_{\xi} F^{i} & =-\sqrt{2} i \bar{\xi}^{i} \gamma^{M} \partial_{M} \chi .
\end{aligned}
$$

To carry out the orientifold projection, we must consistently assign parities $P$ to the various fields and impose the boundary conditions (10). Here is a consistent set of assignments which gives $N=1$ supersymmetry on the wall:

\begin{tabular}{|c|c|c|} 
& $P=+1$ & $P=-1$ \\
\hline \hline$\xi$ & $\xi_{L}^{1}$ & $\xi_{L}^{2}$ \\
\hline$A^{i}$ & $A^{1}$ & $A^{2}$ \\
\hline$\chi$ & $\chi_{L}$ & $\chi_{R}$ \\
\hline $\mathrm{F}^{i}$ & $\mathrm{~F}^{1}$ & $\mathrm{~F}^{2}$ \\
\hline
\end{tabular}

As in Section 2, we project out the odd-parity states and consider the supersymmetry on the boundary generated by $\xi_{L}^{1}$. The transformations (77) specialize to

$$
\begin{aligned}
\delta_{\xi} A^{1} & =\sqrt{2} \xi_{L}^{1 T} \chi_{L} \\
\delta_{\xi} \chi_{L} & =\sqrt{2} i \sigma^{m} \partial_{m} A^{1} \xi_{L}^{1 *}-\sqrt{2} \partial_{5} A^{2} \xi_{L}^{1}+\sqrt{2} F^{1} \xi_{L}^{1} \\
\delta_{\xi} F^{1} & =i \sqrt{2} \xi_{L}^{1 \dagger} \bar{\sigma}^{m} \partial_{m} \chi_{L}+\sqrt{2} \xi_{L}^{1 \dagger} \partial_{5} \chi_{R} \\
\delta_{\xi} \partial_{5} A^{2} & =\sqrt{2} \xi_{L}^{1 \dagger} \partial_{5} \chi_{R} .
\end{aligned}
$$

These transformations imply that

$$
\delta_{\xi}\left(F^{1}-\partial_{5} A^{2}\right)=\sqrt{2} i \xi_{L}^{1 \dagger} \bar{\sigma}^{m} \partial_{m} \chi_{L}
$$

Then $A^{1}, \chi_{L},\left(F^{1}-\partial_{5} A^{2}\right)$ transform as the complex scalar, chiral fermion, and auxiliary field components of a four-dimensional $N=1$ chiral multiplet.

We can use this set of fields to write a coupling of the bulk hypermultiplet to chiral superfields on the boundary. In particular, the boundary theory might have a superpotential which depends on the boundary chiral fields $\phi_{i}$ and the boundary value of the field $A^{1}$. The superpotential term then includes the boundary action

$$
\mathcal{L}_{4}=\left(F^{1}-\partial_{5} A^{2}\right) \frac{d W}{d A^{1}}+\cdots .
$$


If we integrate out the auxiliary field $F^{1}$ and write the resulting action in 5 dimensions, we arrive at the structure

$$
\mathcal{L}_{5}=\left|\partial_{M} A^{2}\right|^{2}-\delta\left(x^{5}\right)\left[\partial_{5} A^{2} \frac{d W}{d A^{1}}+\text { h.c. }\right]+\left(\delta\left(x^{5}\right)\right)^{2}\left|\frac{d W}{d A^{1}}\right|
$$

If we indentify $A^{2}$ with the scalar component of $C_{A B C}$ shown in (76) and $\left(d W / d A^{1}\right)$ with the $E_{8}$ gaugino condensate, this reproduces the perfect-square structure (国) found by Hořava [2, 幽.

From here, we could go on to discuss the communication of supersymmetry breaking. If we simply assume a fixed value of the gaugino condensate and solve for $A^{2}$ as in (33), we find a universal gaugino mass proportional to $1 / \ell$, as in [10, 11, 12]. This leads to conventional supergravity-mediated supersymmetry breaking scenario. It would be very interesting to know whether there are other possibilities. In particular, it would be interesting to find a perturbative hierarchy of soft supersymmetry-breaking terms similar to the one that we discussed in Section 4. To search for such possibilities, it is necessary to understand the general coupling of boundary matter fields to supergravity. We are currently investigating that question.

\section{Conclusion}

In this paper, we have shown how easy it is to construct consistent couplings of fivedimensional supermultiplets to matter multiplets on orientifold walls by analyzing the transformation properties of the associated auxiliary fields. We applied this method to some simple models with bulk and boundary fields and exhibited several possibilities for the communication of supersymmetry breaking from one wall to the other. We hope that this method will generalize to supergravity and allow a more complete understanding of the supersymmetry breaking and its phenomenology in the Hořava-Witten approach to unification.

\section{ACKNOWLEDGEMENTS}

We are grateful to our colleagues at SLAC and Stanford for many discussions of the issues of this paper, and to Michael Dine, Costas Kounnas, Steve Martin, Hans Peter Nilles, Erich Poppitz, Arvind Rajaraman, Lisa Randall, and Leonard Susskind for useful suggestions. This work was supported by the Department of Energy under Contract No. DE-AC03$76 \mathrm{SF} 00515$.

\section{More about the two-loop self-energy}

In this appendix, we will give some further details of the two-loop self-energy calculations discussed in Sections 3 and 4. 
As we explained in (27), our strategy for computing the diagrams shown in Figure 3 began with bringing each diagram into the form

$$
M^{2}=-g^{4} C_{2}(R) C\left(R^{\prime}\right) \int_{q} \int_{k 55} \frac{N\left(k, k^{5}, \hat{k}^{5}, q\right)}{\left(k^{2}\right)\left(k^{2}-\left(k^{5}\right)^{2}\right)\left(k^{2}-\left(\hat{k}^{5}\right)^{2}\right)\left(q^{2}-m_{1}^{2}\right)\left((q-k)^{2}-m_{2}^{2}\right)}
$$

for the $m_{1}, m_{2}$ appropriate to the inner loop of the diagram. We now give the contributions of the various diagrams to the numerator polynomial $N$. In the following formula, we write the contributions to $N$ as a sum, following the order of the diagrams in Figure 3, although properly each separate term should receive the appropriate particle masses in the denominator. The expression is given for the mass spectrum (40) considered in Section 4; for the analysis of Section 3, one should set all masses equal to zero. With this explanation,

$$
\begin{aligned}
N= & 2(k \cdot(2 q-k))^{2}-2\left(q^{2}-m_{+}^{2}+q^{2}-m_{-}^{2}\right) k^{2}-2(2 q-k)^{2} k^{2} \\
& +8\left(q^{2}-m_{+}^{2}+q^{2}-m_{-}^{2}\right) k^{2}+4\left(q \cdot(q-k) k^{2}-2 q \cdot k(q-k) \cdot k-m^{2} k^{2}\right) \\
& -8 k^{2}\left(q \cdot(q-k)-2 m^{2}\right)+16 k^{2} k \cdot(q-k)+2 k^{2}+0+0 .
\end{aligned}
$$

If we set all masses equal to zero, this expression vanishes after the use of the $q \leftrightarrow(k-q)$ symmetry of the denominator. With nonzero masses, some simple rearrangements and a Euclidean rotation bring the expression for $m_{\phi}^{2}$ into the form (44).

In our analysis of (44), we made use of the self-energy integral $b\left(k^{2}, m_{1}^{2}, m_{2}^{2}\right)$ defined by (48). We can write $b$ more explicitly as

$$
\begin{aligned}
b\left(k^{2}, m_{1}^{2}, m_{2}^{2}\right) & =\int_{0}^{\infty} d x \log \left(x(1-x) k^{2}+x m_{1}^{2}+(1-x) m_{2}^{2}\right) \\
& =A \log \left[\frac{\left(A+B_{1}\right)\left(A+B_{2}\right)}{\left(A-B_{1}\right)\left(A-B_{2}\right)}\right]+B_{2} \log m_{1}^{2}+B_{1} \log m_{2}^{2}-2,
\end{aligned}
$$

where

$$
A=\left[\frac{k^{4}+2 k^{2}\left(m_{1}^{2}+m_{2}^{2}\right)+\left(m_{1}^{2}-m_{2}^{2}\right)^{2}}{4 k^{4}}\right]^{1 / 2}
$$

and

$$
B_{1}=\frac{k^{2}+m_{1}^{2}-m_{2}^{2}}{2 k^{2}}, \quad B_{2}=\frac{k^{2}+m_{2}^{2}-m_{1}^{2}}{2 k^{2}} .
$$

From $b\left(k^{2}, m_{1}^{2}, m_{2}^{2}\right)$, we can compute the combinations $R\left(k^{2}\right), S\left(k^{2}\right), P\left(k^{2}\right)$ defined in (49) and (51). We evaluate these expressions using the mass spectrum derived from (40). It is straightforward to work out the asymptotic behavior of these functions for large and small values of $k^{2}$. For $P\left(k^{2}\right)$, we find, as $m^{2} k^{2} \rightarrow 0$,

$$
P\left(k^{2}\right)=k^{2}\left[\frac{4+x-2 x^{2}}{x^{2}} \log (1+x)+1+(x \leftrightarrow-x)\right]+\mathcal{O}\left(k^{4}\right) .
$$

and as $m^{2} k^{2} \rightarrow \infty$,

$$
P\left(k^{2}\right)=\frac{4 m^{4}}{k^{2}}\left[x^{2} \log \frac{k^{2}}{m^{2}}-\left(x^{2}+3 x+2\right) \log (1+x)-x^{2}+(x \leftrightarrow-x)\right]+\mathcal{O}\left(k^{-4}\right) .
$$


The computation of the Casimir energy reported in Section 5 is very similar to the computation of $m_{\phi}^{2}$ and, in particular, uses the same auxiliary function $P\left(k^{2}\right)$.

\section{References}

[1] P. Hořava and E. Witten, Nucl. Phys. B 460, 506 (1996), hep-th/9510209.

[2] P. Hořava and E. Witten, Nucl. Phys. B 475, 94 (1996), hep-th/9603142.

[3] E. Witten, Nucl. Phys. B 471, 135 (1996), hep-th/9602070.

[4] P. Hořava, Phys. Rev. D 54, 7561 (1996), hep-th/9608019.

[5] E. Sharpe, hep-th/9611196.

[6] H. G. B. Casimir, Proc. Kong. Ned. Akad. Wet. B 51, 793 (1948).

[7] T. Banks and M. Dine, Nucl. Phys. B 479, 173 (1996), hep-th/9605136.

[8] I. Antoniadis, S. Ferrara, and T. R. Taylor, Nucl. Phys. B 460, 489 (1996), hepth/9511108.

[9] K. Choi, Phys. Rev. D 56, 6588 (1997), hep-th/9706171.

[10] T.-J. Li, J. L. Lopez, and D. V. Nanopoulos, Phys. Rev. D 56, 2602 (1997), hepph/9704247.

[11] H. P. Nilles, M. Olechowski, and M. Yamaguchi, Phys. Lett. B 415, 24 (1997), hepth/9707143.

[12] Z. Lalak and S. Thomas, Nucl. Phys. B 515, 55 (1998), hep-th/9707223.

[13] K. Choi, H. B. Kim, and C. Munoz, hep-th/9711158.

[14] A. Lukas, B. A. Ovrut, and D. Waldram, hep-th/9711197.

[15] M. Dine, R. Rohm, N. Seiberg, and E. Witten, Phys. Lett. B 156, 55 (1985).

[16] E. Dudas and C. Grojean, Nucl. Phys. B 507, 553 (1997), hep-th/9704177.

[17] I. Antoniadis and M. Quirós, Nucl. Phys. B 505, 109 (1997), hep-th/9705037; I. Antoniadis and M. Quirós, Phys. Lett. B 416, 327 (1998), hep-th/9707208.

[18] J. Scherk and J. H. Schwarz, Phys. Lett. B 82, 60 (1979).

[19] P. Brax and N. Turok, Phys. Lett. B 413, 331 (1997), hep-th/9706035. 
[20] M. Dine, A. E. Nelson, and Y. Shirman, Phys. Rev. D 51, 1362 (1995), hep-ph/9408384;

M. Dine, A. E. Nelson, Y. Nir, and Y. Shirman, Phys. Rev. D 53, 2658 (1996), hepph/9507378.

[21] S. Martin, Phys. Rev. D 55, 3177 (1997), hep-ph/9608224.

[22] E. Poppitz and S. P. Trivedi, Phys. Lett. B 401, 38 (1997), hep-ph/9703246.

[23] J. van der Bij and M. Veltman, Nucl. Phys. B 231, 205 (1984).

[24] G. F. Giudice and R. Rattazzi, Nucl. Phys. B 511, 25 (1998), hep-ph/9706540.

[25] S. Dimopoulos, G. F. Giudice, and A. Pomerol, Phys. Lett. B389, 37 (1996), hepph/9607225.

[26] P. West, Introduction to Supersymmetry and Supergravity. (World Scientific, Singapore, 1986).

[27] J. Wess and J. Bagger, Supersymmetry and Supergravity. (Princeton University Press, Princeton, 1992). 